Research Paper

\title{
Segmentectomy or Wedge Resection in Stage IA Lung Squamous Cell Carcinoma and Adenocarcinoma?
}

\author{
Guoshu $\mathrm{Li}^{1 \#}$, Shuanshuan Xie ${ }^{1 \#}$, Feng $\mathrm{Hu}^{2 \#}$, Min Tan ${ }^{1}$, Lihong Fan ${ }^{\circledR}$ and Changhui Wang ${ }^{1 凶}$ \\ 1. Department of Respiratory Medicine, Shanghai Tenth People's Hospital, Tongji University School of Medicine, Shanghai 200072, China. \\ 2. Tongren Hospital, Shanghai Jiao Tong University School of Medicine, 1111 XianXia Road, Shanghai 200336, China. \\ \#These authors have contributed equally to this work.
}

$\triangle$ Corresponding author: Lihong Fan, Changhui Wang. Department of Respiratory Medicine, Shanghai Tenth People's Hospital, Tongii University School of Medicine, No. 301, Yanchang Middle Road, Jing'an District, Shanghai 200072, People's Republic of China. E-mail: fan-li-hong@Outlook.com; wang-chang-hui@hotmail.com; Fax number: 86-021-66301685; Telephone: 86-021-66301685.

() The author(s). This is an open access article distributed under the terms of the Creative Commons Attribution License (https://creativecommons.org/licenses/by/4.0/). See http://ivyspring.com/terms for full terms and conditions.

Received: 2020.06.18; Accepted: 2020.12.24; Published: 2021.01.18

\begin{abstract}
Objectives: We performed this study to compare survival outcomes of segmentectomy (SG) and wedge resection (WR) in stage IA lung squamous cell carcinoma (SQCC) and lung adenocarcinoma (AD).

Methods: Using the Surveillance, Epidemiology, and End Results registry (SEER), we identified 1529 and 4070 patients with stage IA SQCC and AD, respectively, who had complete clinical information between 2004 and 2015. We used Kaplan-Meier analysis to determine the propensity score for patients with limited resection based on the preoperative characteristics of patients. Lung cancer-specific survival (LCSS) was compared in patients treated with WR and SG after adjusting, stratifying, or matching lung cancer patients according to propensity score.

Results: Kaplan-Meier analysis demonstrated that there was a statistically significant difference in survival curves (log rank $P=0.01$ ) for patients with stage IA SQCC between SG and WR. But there was no statistically significant difference in survival curves (log rank $P>0.05$ ) in patients with stage IA AD between the two limited resections. Compared with the WR, The hazard ratios ( $95 \%$ confidence intervals) of SG were 0.689 (0.519-0.914) and $0.896(0.752-1.067)$ in patients with stage IA SQCC and AD, respectively.

Conclusion: This study suggests that SG can yield superior survival outcome compared with WR in patients with stage IA SQCC. However, the survival outcomes of SG and WR are generally equivalent in patients with stage IA AD.
\end{abstract}

Key words: SEER, segmentectomy, wedge resection, non-small cell lung cancer, survival

\section{Introduction}

Lung cancer is a serious threat to human health, and its incidence has risen rapidly in recent years. Patients with advanced non-small cell lung cancer (NSCLC) have a poor prognosis, but those with stage IA NSCLC have relatively good long-term outcomes after appropriate treatment [1]. Some studies have reported that the 5-year overall survival rate of patients with stage IA NSCLC after surgery is more than $70 \%$, and the prognosis is even better for patients with tumor sizes $\leq 2 \mathrm{~cm}$ [2-5]. Although most patients with NSCLC are diagnosed at an advanced stage, $10 \%-15 \%$ of them are diagnosed with stage IA NSCLC [1]. Moreover, with the introduction of highresolution computed tomography (CT) and low-dose helical CT screening for lung cancer, the number of patients diagnosed with early-stage lung cancer has increased [6]. Surgery is one of the most important treatment methods for stage IA NSCLC. Currently, lobectomy remains the standard surgical treatment for patients with NSCLC. However, limited resection is commonly used to treat patients who are unable to undergo total lobectomy due to older age, severe impairment of lung function, or other comorbidities [7]. In recent years, many studies have shown that the survival rate of patients with stage IA NSCLC who undergo limited resection is similar to that of patients who undergo lobectomy [8-13]. In addition, patients who undergo limited resection have less resected lung 
tissue and greater retention of lung function than those who undergo conventional lobectomy, which provides an opportunity for subsequent surgery if a second primary lung cancer occurs [14]. Therefore, limited resection has become an important treatment for patients with stage IA NSCLC.

Limited resection methods include wedge resection (WR) and segmentectomy (SG). SG, as an anatomic resection, has been thought as a preferred approach compared to WR in patients with stage IA NSCLC [15]. However, due to the quality of life in patients receiving SG was not as good as patients undergoing WR $[16,17]$, the option of WR or SG should be more cautious for patients. Previous studies have compared survival rates between the two types of limited resection primarily based on variables such as tumor size, differentiation grade, age, and so on, but less on the pathology subtypes of NSCLC. This study was to compare survival outcomes of WR and SG by comparing with lung cancer-specific survival (LCSS) in patients with stage IA lung squamous cell carcinoma (SQCC) or lung adenocarcinoma (AD), the major pathology subtypes of NSCLC.

\section{Methods}

\section{Data source}

This retrospective study was conducted to assess the relationship between two types of limited resection and the survival rate in patients with IA stage SQCC or AD, using data from the Surveillance, Epidemiology, and End Results (SEER) database. The SEER project is maintained by the National Cancer Institute in the United States (US). SEER includes a population-based cancer registry, established in 1973, which accounts for approximately $10 \%$ of the US population [18].

\section{Study population}

We limited the cohort to patients with stage IA SQCC or $\mathrm{AD}(\leq 3 \mathrm{~cm}$ in tumor size) diagnosed between 2004 and 2015. All included patients underwent WR or SG; the complete information of all included patients was available in the SEER database. Figure 1 shows a flow chart of the literature search for analyses based on the SEER database. Initially, 68,282 patients with IA stage lung cancer were identified. A total 66,312 patients with NSCLC were included, after excluding the following patients: 1,185 patients with small cell lung cancer, 66 with carcinoma not otherwise specified (NOS), 179 with large cell carcinoma, 371 with adenosquamous carcinoma and 169 patients with other cancers. We included 21,385 patients with NSCLC who were treated surgically, after excluding the following patients: 191 patients who underwent unknown surgery; 23,493 with no surgery; 9 with local tumor destruction or excision NOS; 120 with surgery NOS; 509 with laser ablation, cryosurgery, or radiofrequency ablation; 20,459 who underwent lobectomy with mediastinal lymph node dissection, plus pleura or diaphragm; and 146 patients with excision or resection of less than one lobe NOS. Finally, a total of 5,599 patients with stage IA SQCC or $\mathrm{AD}$ who were treated with limited resection were included in the analysis, after excluding 15,786 patients with NA not the first tumor.

\section{Clinicopathological data}

According to histologic type, histologic codes were classified as follows: (1) SQCC: 8052, 8070-8075, $8083,8084,8123$, (2) AD: 8244, 8245, 8250-8255, 8260, $8290,8310,8323,8333,8480,8481,8490,8507,8550$, $8570,8571,8574$, and 8576. Based on information of the SEER site-specific surgical variables, all 5,599 included patients with stage IA SQCC or AD were classified as having undergone WR (SEER surgical code 21) or SG (SEER surgical code 22).

\section{Statistical analyses}

Classification variables were compared using the Pearson's chi-squared test. Kaplan-Meier analysis and the log-rank test were used to compare survival between the WR group and the SG group. Propensity score methods were used to control the potential differences in baseline characteristics of the included patients. Cox regression was performed to assess whether the baseline covariates of the two groups were balanced after adjusting for the estimated propensity scores. Statistical significance was set at a two-tailed $P$ value $<0.05$. Data were analyzed using IBM SPSS version 20.0 (IBM Corp, Armonk, NY, USA).

\section{Results}

\section{Study cohort characteristics}

We identified 5,599 limited resection patients with stage IA SQCC or AD, of whom 4,394 (78.5\%) and 1,205 (21.5\%) underwent WR and SG, respectively, as a primary treatment from 2004 to 2015. Table 1 shows the baseline characteristics of all patients. Kaplan-Meier analyses demonstrated that there were no statistically significant differences in LCSS with regard to tumor location $(P=0.996)$, marital status $(P=0.482)$, laterality $(P=0.854)$ and high school education $(P=0.079)$ between the two groups. However, significant differences in LCSS were found with respect to age $(P<0.001)$, sex $(P<0.001)$, tumor size $(P<0.001)$, differentiation grade $(P<0.001)$, histologic type $(P<0.001)$, radiotherapy $(P<0.001)$, and chemotherapy $(P<0.001)$ (Table 1$)$. 
Table 1. Baseline characteristics of patients with stage IA non-small cell lung cancer treated with limited resection in the Surveillance, Epidemiology and End Results (SEER) program, 2004-2015

\begin{tabular}{|c|c|c|c|c|c|}
\hline \multirow[t]{2}{*}{ Characteristics } & \multicolumn{2}{|c|}{ Wedge resection } & \multicolumn{2}{|c|}{ Segmentectomy } & \multirow[t]{2}{*}{$P$} \\
\hline & Number & $\%$ & Number & $\%$ & \\
\hline Age, year & & & & & $<0.001$ \\
\hline$<45$ & 35 & 0.8 & 12 & 1.0 & \\
\hline$\geq 45,<55$ & 271 & 6.2 & 72 & 6.0 & \\
\hline$\geq 55,<65$ & 950 & 21.6 & 276 & 22.9 & \\
\hline$\geq 65,<75$ & 1734 & 39.5 & 487 & 40.4 & \\
\hline$\geq 75$ & 1404 & 31.9 & 358 & 29.7 & \\
\hline Sex & & & & & $<0.001$ \\
\hline Female & 2462 & 56.0 & 714 & 59.3 & \\
\hline Male & 1932 & 44.0 & 491 & 40.7 & \\
\hline Tumor size, cm & & & & & $<0.001$ \\
\hline$\leq 1$ & 893 & 20.3 & 167 & 13.9 & \\
\hline$>1, \leq 2$ & 2484 & 56.5 & 697 & 57.8 & \\
\hline$>2, \leq 3$ & 1017 & 23.2 & 341 & 28.3 & \\
\hline Tumor location & & & & & 0.996 \\
\hline Upper lobe & 2837 & 64.5 & 707 & 58.6 & \\
\hline Middle lobe & 178 & 4.1 & 26 & 2.2 & \\
\hline Lower lobe & 1331 & 30.3 & 465 & 38.6 & \\
\hline Not otherwise specified & 37 & 0.8 & 5 & 0.4 & \\
\hline Overlapping lesion & 11 & 0.3 & 2 & 0.2 & \\
\hline Differentiation grade & & & & & $<0.001$ \\
\hline Well differentiated & 1054 & 24.0 & 278 & 23.1 & \\
\hline Moderately differentiated & 1886 & 42.9 & 575 & 47.7 & \\
\hline Poorly differentiated & 1081 & 24.6 & 263 & 21.8 & \\
\hline Undifferentiated & 373 & 8.5 & 89 & 7.4 & \\
\hline Laterality & & & & & 0.854 \\
\hline Right-origin of primary & 2499 & 56.9 & 619 & 51.4 & \\
\hline Left origin of primary & 1895 & 43.1 & 586 & 48.6 & \\
\hline Histologic type & & & & & $<0.001$ \\
\hline Adenocarcinoma & 3163 & 72.0 & 907 & 75.3 & \\
\hline Squamous cell carcinoma & 1231 & 28.0 & 298 & 24.7 & \\
\hline Radiotherapy & & & & & $<0.001$ \\
\hline Yes & 223 & 5.1 & 36 & 3.0 & \\
\hline No & 4143 & 94.3 & 1165 & 96.7 & \\
\hline Others & 28 & 0.6 & 4 & 0.3 & \\
\hline Chemotherapy & & & & & $<0.001$ \\
\hline Yes & 170 & 3.9 & 36 & 3.0 & \\
\hline No & 4224 & 96.1 & 1169 & 97.0 & \\
\hline Marital status & & & & & 0.482 \\
\hline Married & 2363 & 53.8 & 680 & 56.4 & \\
\hline Single & 453 & 10.3 & 137 & 11.4 & \\
\hline Divorced & 592 & 13.5 & 135 & 11.2 & \\
\hline Widowed & 981 & 22.3 & 251 & 20.8 & \\
\hline $\begin{array}{l}\text { Unmarried or domestic } \\
\text { partner }\end{array}$ & 5 & 0.1 & 2 & 0.2 & \\
\hline High school education & & & & & 0.079 \\
\hline$\geq 21$ & 793 & 18.0 & 244 & 20.2 & \\
\hline $13-20$ & 1304 & 29.7 & 279 & 23.2 & \\
\hline $7-12.9$ & 1963 & 44.7 & 567 & 47.1 & \\
\hline$<7$ & 334 & 7.6 & 115 & 9.5 & \\
\hline
\end{tabular}

Subsequent analysis using a Cox model, which included above seven significant covariates, showed that there were no statistically significant differences for histologic type $(P=0.263)$ in patients with stage IA SQCC or AD. However, there were statistically significant differences for age $(P<0.001)$, sex $(P<0.001)$, tumor size $(P<0.001)$, differentiation grade $(P<0.001)$, radiotherapy $(P<0.001)$, chemotherapy $(P<0.001)$, and resection $(P=0.013) \quad($ Table 2$)$. These outcomes demonstrate that the prognoses of patients with stage IA SQCC or AD are related to the followed factors: age, sex, tumor size, differentiation grade, radiotherapy, chemotherapy, and limited resection, but are not related to these factors: tumor location, marital status and laterality.

Table 2. Multivariate analysis using a cox proportional hazards model in patients with stage IA non-small cell lung cancer

\begin{tabular}{|c|c|c|c|}
\hline \multirow[t]{2}{*}{ Variable } & \multicolumn{3}{|c|}{ Multivariate analysis } \\
\hline & HR & $95 \% \mathrm{CI}$ & $P$ \\
\hline \multicolumn{4}{|l|}{ Sex } \\
\hline Female & Reference & & \\
\hline Male & 1.296 & 1.152 to 1.457 & $<0.001$ \\
\hline Differentiation grade & & & $<0.001$ \\
\hline Well differentiated & Reference & & \\
\hline Moderately differentiated & 1.644 & 1.377 to 1.964 & $<0.001$ \\
\hline Poorly differentiated & 1.929 & 1.592 to 2.339 & $<0.001$ \\
\hline Undifferentiated & 1.568 & 1.216 to 2.023 & 0.001 \\
\hline \multicolumn{4}{|l|}{ Histologic type } \\
\hline Adenocarcinoma & Reference & & \\
\hline Squamous cell carcinoma & 1.077 & 0.945 to 1.228 & 0.263 \\
\hline \multicolumn{4}{|l|}{ Limited resection } \\
\hline Wedge resection & Reference & & \\
\hline Segmentectomy & 0.827 & 0.712 to 0.960 & 0.013 \\
\hline Radiotherapy & & & $<0.001$ \\
\hline Yes & Reference & & \\
\hline No & 0.648 & 0.520 to 0.807 & $<0.001$ \\
\hline Others & 1.166 & 0.585 to 2.322 & 0.662 \\
\hline \multicolumn{4}{|l|}{ Chemotherapy } \\
\hline Yes & Reference & & \\
\hline No & 0.566 & 0.452 to 0.709 & $<0.001$ \\
\hline Tumor size, cm & & & $<0.001$ \\
\hline$\leq 1$ & Reference & & \\
\hline$>1, \leq 2$ & 1.247 & 1.045 to 1.488 & 0.014 \\
\hline$>2, \leq 3$ & 1.685 & 1.393 to 2.039 & $<0.001$ \\
\hline Age, year & & & $<0.001$ \\
\hline$<45$ & Reference & & \\
\hline$\geq 45,<55$ & 1.892 & 0.757 to 4.728 & 0.172 \\
\hline$\geq 55,<65$ & 1.875 & 0.772 to 4.554 & 0.165 \\
\hline$\geq 65,<75$ & 2.048 & 0.846 to 4.959 & 0.112 \\
\hline$\geq 75$ & 2.975 & 1.228 to 7.208 & 0.016 \\
\hline
\end{tabular}

Table 3. Univariate analysis comparing HR (SG vs. WR) in patients

\begin{tabular}{lllll}
\hline Variable & Number & \multicolumn{4}{l}{ Univariate Analysis } \\
\cline { 3 - 5 } & & HR & $95 \%$ CI & $P$ \\
\hline Total & 5599 & 0.823 & 0.710 to 0.955 & 0.010 \\
SQCC & 1529 & 0.689 & 0.519 to 0.914 & 0.010 \\
AD & 4070 & 0.896 & 0.752 to 1.067 & 0.217 \\
\hline
\end{tabular}

Abbreviations: SQCC, squamous cell carcinoma; AD, adenocarcinoma; SG,

segmentectomy; WR, wedge resection; $\mathrm{HR}$, hazard ratio; $\mathrm{CI}$, confidence interval.

Table 3 shows the hazard ratio (HR) and 95\% confidence interval $(\mathrm{CI})$ between the WR and SG. Compared with the WR group, the HR $(95 \% \mathrm{CI}, P)$ of the SG group were $0.823(0.710-0.955, P=0.01)$ in total patients. The HR $(95 \% \mathrm{CI}, P)$ of the SG group were 0.689 (0.519-0.914, $P=0.01), 0.896$ (0.752-1.067, $P=0.217)$ comparing with the WR group in patients with SQCC 
and $\mathrm{AD}$, respectively. These outcomes demonstrate that the comparative results of $\mathrm{WR}$ and $\mathrm{SG}$ are different in pathology subtypes of NSCLC: the SG yields better survival outcome than the WR in patients with stage IA SQCC, but the survival outcomes of SG and WR are generally equivalent in patients with stage IA AD.

\section{Comparison of survival curves between WR and SG}

According to Kaplan-Meier analysis, the survival curves were compared between WR and SG groups (Figure 2). The survival curve of the SG group is better than the $W R$ group $(\log$ rank $P=0.01)$ in total patients (Figure 2A). Similarly, the survival curve of the SG group is better (log rank $P=0.01)$ than the WR group in patients with stage IA SQCC (Figure 2B). However, in patients with stage IA AD, the survival curves (log rank $P=0.217$ ) show no statistically significant difference between the WR and SG groups (Figure $2 \mathrm{C})$. This outcome also demonstrates that the SG can yield better survival outcome than WR in patients with stage IA SQCC, but the SG and WR yield generally equivalent survival outcomes in patients with stage IA AD.

\section{Comparison of lung cancer-specific mortality between the WR and SG groups}

The lung cancer-specific mortality was 21.4 (940/4394) for WR group, and $17.8 \%$ (214/1205) for SG group in patients with stage IA NSCLC. The lung cancer-specific mortalities were $26.0 \%(320 / 1231)$ and $19.10 \%(249 / 1304)$ for the WR and SG groups in patients with stage IA SQCC, respectively. In patients with stage IA AD, The lung cancer-specific mortalities were $19.6 \%(620 / 3163)$ and $17.3 \%(157 / 907)$ for the WR and SG groups, respectively (Table 4). The outcomes indicate that the lung cancer-specific mortality of SG group is superior to WR group in stage IA SQCC, but this advantage decreases obviously in stage IA AD.

Table 4. Lung cancer-specific mortality, median survival time and mean survival time of WR and SG in different histologic types (SEER database, 2004-2015)

\begin{tabular}{lllllll}
\hline $\begin{array}{l}\text { Histologic } \\
\text { type }\end{array}$ & \multicolumn{2}{l}{ Mortality n/N (\%) } & \multicolumn{2}{c}{$\begin{array}{l}\text { Median survival } \\
\text { time (months) }\end{array}$} & \multicolumn{2}{l}{$\begin{array}{l}\text { Mean survival } \\
\text { time (months) }\end{array}$} \\
\cline { 2 - 7 } & WR & SG & WR & SG & WR & SG \\
\hline NSCLC & $21.4(940 / 4394)$ & $17.8(214 / 1205)$ & 38.00 & 37.00 & 44.56 & 45.02 \\
SQCC & $26.0(320 / 1231)$ & $19.1(57 / 298)$ & 34.00 & 34.00 & 41.11 & 44.24 \\
AD & $19.6(620 / 3163)$ & $17.3(157 / 907)$ & 39.00 & 38.00 & 45.90 & 45.28 \\
\hline Abbreviations: NSCLC, non-small cell lung cancer; SQCC, squamous cell \\
\multicolumn{2}{l}{ carcinoma; AD, adenocarcinoma; WR, wedge resection; SG, segmentectomy. }
\end{tabular}

xcluded

Small cell lung cancer $(n=1,185)$

Carcinoma, NOS $(n=66)$

Large cell carcinoma $(n=179)$

Adenosquamous carcinoma $(n=371)$

Others $(n=169)$

IA NSCLC patients

Year of diagnosis, 2004-2015 $(\mathrm{N}=66,312)$

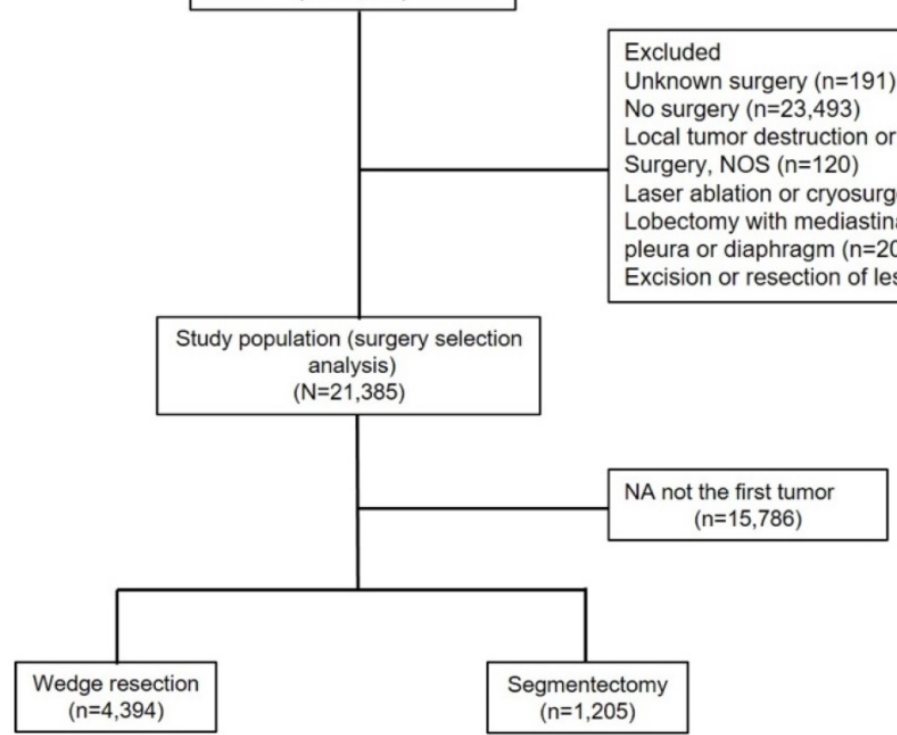

Figure 1. Study flow diagram. Abbreviations: NSCLC, non-small cell lung cancer; NOS, not otherwise specified; RFA, radiofrequency ablation. 

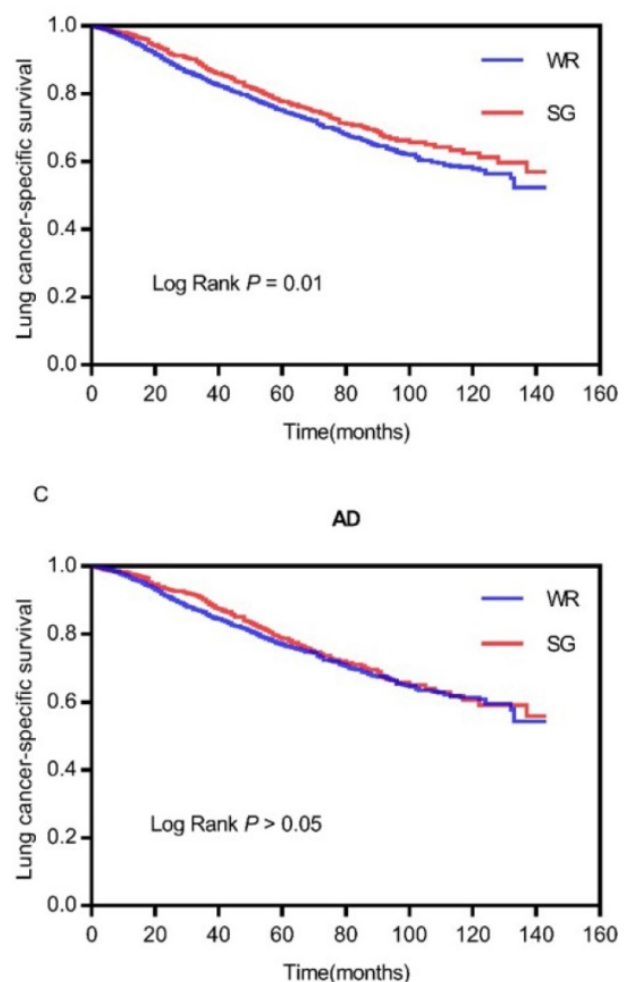

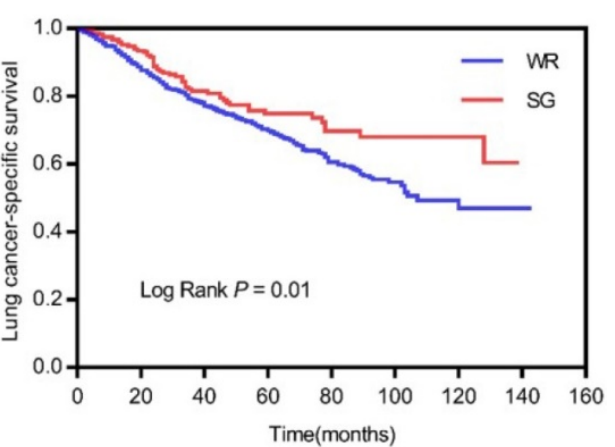

Figure 2. Comparison of survival curves between WR and SG. (A) Comparison of survival curves ( $P=0.01)$ in patients with stage IA NSCLC; (B) Comparison of survival curves $(P=0.01)$ in patients with stage IA SQCC; $(C)$ Comparison of survival curves $(P>0.05)$ in patients with stage IA AD. Abbreviations: LCSS, lung cancer-specific survival; NSCLC, non-small cell lung cancer; SQCC, squamous cell carcinoma; $A D$, adenocarcinoma.

Table 4 also shows the median survival time and the mean survival time of WR and SG groups in different histologic types. In total NSCLC patients, the median survival time and the mean survival time were 38.0 months and 44.56 months for WR group, and 37.00 months and 45.02 months for SG group. In SQCC patients, the median survival time and the mean survival time were 34.00 months and 41.11 months for WR group, and 34.00 months and 44.24 months for SG group. In AD patients, the median survival time and the mean survival time were 39.00 months and 45.90 months for WR group, and 38.00 months and 45.28 months for SG group. The datum demonstrated that the difference of mean survival time is more obvious in SQCC than AD between WR and SG groups.

\section{Discussion}

Currently, with advances in radiology and the emergence of lung cancer screening programs, a higher incidence of early stage NSCLC has been observed. In this scenario, limited resection has been adopted as an effective treatment for stage IA NSCLC patients, especially in the elderly and patients with pulmonary insufficiency. Over the past decade, SG has become more common and played an increasingly important role in the treatment of pulmonary metastases. It is technically difficult for anatomic SG which not only demands a thorough understanding of the pulmonary anatomy, but also requires in-depth tomographic study of the location of the pulmonary nodules. Persistent pneumothorax (over five days) is the most common complication of SG, which occurs between $8 \%$ and $10 \%$ [19]. Therefore, the nodules must be well positioned to ensure safe resection. WR has been considered "inferior" cancer operation for nearly two decades, but much of the contemporary literature is contradictory and inconclusive. Several recent studies have shown that WR is even equivalent to lobectomy [20-22]. There is enough controversy to avoid overly dogmatic statements about the poor quality of WR in the absence of modern large-scale prospective trials. Instead, the surgeon should place the emphasis on the quality of the operation and ensure the most possible excision of margin when WR is required. Performed well, WR is maybe an appropriate surgical option.

Some researches has focused on which limited resection was better to the survival of NSCLC patients. Many people have reported that SG is superior to WR for NSCLC patients. For example, Dai et al. [23] considered that SG should be recommended for patients with NSCLC in whom lobectomy is unsuitable. Hou et al. [24] reported that SG results in 
higher survival rates than WR in patients with stage I NSCLC. Reveliotis et al.[25] reported that SG is superior to WR in terms of local recurrence and cancer-related mortality rates, and those authors recommended SG for high-risk patients. However, there were some different sounds about this view. In 2016, Altorki et al. [26] reported a retrospective analysis and concluded that WR maybe ontologically equivalent to anatomic SG for clinical T1a tumors. Sybron Harrison et al. [22] has reported a contemporary prospective randomized trial (ACOSOG Z4032) which supported to the view that WR and SG maybe equivalent methods of sub-lobar resection. In our study, we evaluated the survival outcomes of SG versus WR in patients with stage IA NSCLC. Although we found that SG yielded better survival rate than WR in overall patients, there were different in pathology subtypes of NSCLC. The result shows that SG yields better survival rate than WR in patients with stage IA SQCC, but SG and WR yield generally equivalent survival rate in patients with stage IA AD. In addition, patients underwent SG can obtain more advantageous mean survival time than WR in patients with stage IA SQCC, but this advantage almost lost in patients with stage IA AD. This result may provide patients with stage IA AD a personalized surgical option.

Admittedly, there are some limitations in our study, mainly owing to its retrospective design. For example, the lack of original datum from our own studies as well as validation for main findings and conclusion. In addition, the SEER database does not include some important information, such as the types of therapy, histological subtypes, and gene mutations. This information should be included in future prospective studies. Nevertheless, with the inclusion of 12 variables and nearly 5,600 patients in our cohort, the present study represents a wellbalanced analysis of SG and WR surgical methods. Thus, in the absence of data from prospective trials, our findings can provide information that is useful for the management of patients with stage IA SQCC and AD.

In summary, the prognosis of patients with stage IA NSCLC is related to a variety of factors.

According to the development trend of modern medicine, there must be a personalized surgical approach which should take into account the characteristics of each patient, the imaging characteristics of tumor as well as the impact on the quality of life and surgical recovery in the future. This study indicates that SG is superior to $W R$ in patients with stage IA SQCC, but the survival outcomes of SG and WR are generally equivalent in patients with stage IA AD. The result may provide a basis for individualized surgical option for stage IA $\mathrm{AD}$ patients.

\section{Abbreviations}

NSCLC, non-small cell lung cancer; WR, wedge resection; SG, segmentectomy; SEER, Surveillance, Epidemiology and End Results; LCSS, lung cancerspecific survival; SQCC, squamous cell carcinoma; $\mathrm{AD}$, adenocarcinoma; NOS, not otherwise specified; $\mathrm{HR}$, hazard ratio; $\mathrm{CI}$, confidence interval.

\section{Acknowledgements}

We would like to thank all the staff of the National Cancer Institute for their efforts in the SEER program.

\section{Funding}

This work was supported by the National Natural Science Foundation of China (No. 81802262) and Shanghai Tenth Hospital's improvement plan for NSFC (No. 04.03.17.032, 04.01.18. 048).

\section{Author Contributions}

Conception and design: G.S.L. and S.S.X. Acquisition, statistical analysis or interpretation of the data: all authors. Drafting of the manuscript: L.H.F. and C.H.W. All authors reviewed and approved the final version of the manuscript.

\section{Ethical approval}

All procedures performed in studies involving human participants were in accordance with the ethical standards of the institutional and/or national research committee and with the 1964 Declaration of Helsinki and its later amendments or comparable ethical standards. For this type of study, formal consent is waived.

\section{Competing Interests}

The authors have declared that no competing interest exists.

\section{References}

1. Spiro SG, Porter JC. Lung cancer--where are we today? Current advances in staging and nonsurgical treatment. Am J Respir Crit Care Med. 2002; 166:1166-1196.

2. Nesbitt JC, Putnam JB Jr, Walsh GL, et al. Survival in early-stage non-small cell lung cancer. Ann Thorac Surg. 1995; 60:466-472.

3. Williams DE, Pairolero PC, Davis CS, et al. Survival of patients surgically treated for stage I lung cancer. J Thorac Cardiovasc Surg. 1981; 82:70-76.

4. Harpole DH Jr, Herndon JE 2nd, Young WG Jr, et al. Stage I non-small cell lung cancer. A multivariate analysis of treatment methods and patterns of recurrence. Cancer. 1995; 76:787-796.

5. Martini N, Bains MS, Burt ME, et al. Incidence of local recurrence and second primary tumors in resected stage I lung cancer. J Thorac Cardiovasc Surg. 1995; 109:120-129.

6. Xue W, Duan G, Zhang X, et al. Meta-analysis of segmentectomy versus wedge resection in stage IA non-small-cell lung cancer. Onco Targets Ther. 2018; 11:3369-3375.

7. Wisnivesky JP, Henschke CI, Swanson S, et al. Limited resection for the treatment of patients with stage IA lung cancer. Ann Surg. 2010; 251: 550-554. 
8. Zhong C, Fang W, Mao T, et al. Comparison of thoracoscopic segmentectomy and thoracoscopic lobectomy for small-sized stage IA lung cancer. Ann Thorac Surg. 2012; 94: 362-367.

9. Watanabe T, Okada A, Imakiire T, et al. Intentional limited resection for small peripheral lung cancer based on intraoperative pathologic exploration. Jpn J Thorac Cardiovasc Surg. 2005; 53: 29-35.

10. Tsutani Y, Miyata Y, Nakayama H, et al. Oncologic outcomes of segmentectomy compared with lobectomy for clinical stage IA lung adenocarcinoma: propensity score-matched analysis in a multicenter study. J Thorac Cardiovasc Surg. 2013; 146:358-364.

11. Okada M, Koike T, Higashiyama $M$, et al. Radical sublobar resection for small-sized non-small cell lung cancer: a multicenter study. J Thorac Cardiovasc Surg. 2006; 132:769-775.

12. Koike $\mathrm{T}$, Yamato $\mathrm{Y}$, Yoshiya $\mathrm{K}$, et al. Intentional limited pulmonary resection for peripheral T1 N0 M0 small-sized lung cancer. J Thorac Cardiovasc Surg. 2003; 125:924-928.

13. Sugi K, Kobayashi S, Sudou M, et al. Longterm prognosis of video-assisted limited surgery for early lung cancer. Eur J Cardiothorac Surg. 2010; 37:456-460.

14. Smith $\mathrm{CB}$, Swanson SJ, Mhango G, et al. Survival after segmentectomy and wedge resection in stage I non-small-cell lung cancer. J Thorac Oncol. 2013; 8:73-78.

15. Sihoe AD, Van Schil P. Non-small cell lung cancer: when to offer sublobar resection. Lung Cancer. 2014; 86:115-120.

16. Fernando HC, Landreneau RJ, Mandrekar SJ, et al. Analysis of longitudinal quality-of-life data in high-risk operable patients with lung cancer: results from the ACOSOG Z4032 (Alliance) multicenter randomized trial. J Thorac Cardiovasc Surg. 2015; 149:718-725; discussion 725-726.

17. Chen H, Louie AV. SABR vs. Limited Resection for Non-small Cell Lung Cancer: Are We Closer to an Answer? Curr Treat Options Oncol. 2016; 17: 27.

18. Yang J, Zhang $Y$, Sun $X$, et al. The prognostic value of multiorgan metastases in patients with non-small cell lung cancer and its variants: a SEER-based study. J Cancer Res Clin Oncol. 2018; 144:1835-1842.

19. Zhao K, Mei J, Xia C, et al. Prolonged air leak after video-assisted thoracic surgery lung cancer resection: risk factors and its effect on postoperative clinical recovery. J Thorac Dis. 2017; 9:1219-1225.

20. Altorki NK, Yip R, Hanaoka T, et al. Sublobar resection is equivalent to lobectomy for clinical stage 1A lung cancer in solid nodules. J Thorac Cardiovasc Surg. 2014; 147:754-764.

21. Razi SS, Daskalaki D, Burack J. Current trends in lung resection for T1a non-small cell lung cancer: is lobectomy still the answer? J Thorac Dis. 2017; 9:E164-E165.

22. Harrison S, Stiles B, Altorki N. What is the role of wedge resection for T1a lung cancer? J Thorac Dis. 2018;10(Suppl 10):S1157-S1162.

23. Dai C, Shen J, Ren Y, et al. Choice of Surgical Procedure for Patients With Non-Small-Cell Lung Cancer $\leq 1 \mathrm{~cm}$ or $>1$ to $2 \mathrm{~cm}$ Among Lobectomy, Segmentectomy, and Wedge Resection: A Population-Based Study. J Clin Oncol. 2016; 34:3175-3182.

24. Hou B, Deng XF, Zhou D, et al. Segmentectomy versus wedge resection for the treatment of high-risk operable patients with stage I non-small cell lung cancer: a meta-analysis. Ther Adv Respir Dis. 2016; 10:435-443.

25. Reveliotis K, Kalavrouziotis G, Skevis $\mathrm{K}$, et al. Wedge resection and segmentectomy in patients with stage I non-small cell lung carcinoma. Oncol Rev. 2014; 8:74-81.

26. Altorki NK, Kamel MK, Narula N, et al. Anatomical segmentectomy and wedge resections are associated with comparable outcomes for patients with small ct1n0 non-small cell lung cancer. J Thorac Oncol. 2016; 11:1984-1992. 